University of Wollongong

Research Online

Faculty of Engineering and Information

Faculty of Engineering and Information

Sciences - Papers: Part A

Sciences

$1-1-2013$

\title{
Practical fabrication and analysis of an optimized compact eight-channel silicon arrayed-waveguide grating
}

Hongqiang Li

Tianjin Polytechnic University, lihongqiang@tjpu.edu.cn

Yaoting Bai

Tianjin Polytechnic University

Xiaye Dong

Tianjin Polytechnic University

Enbang Li

University of Sydney, enbang@uow.edu.au

Yang Li

Tianjin Polytechnic University

See next page for additional authors

Follow this and additional works at: https://ro.uow.edu.au/eispapers

Part of the Engineering Commons, and the Science and Technology Studies Commons

Research Online is the open access institutional repository for the University of Wollongong. For further information contact the UOW Library: research-pubs@uow.edu.au 


\title{
Practical fabrication and analysis of an optimized compact eight-channel silicon arrayed-waveguide grating
}

\author{
Abstract \\ We have designed, fabricated, and characterized a $1 \times 8$ ultrasmall compact arrayed-waveguide grating \\ (AWG) on silicon-on-insulator (SOI) in a fiber grating demodulation integration microsystem. The \\ miniature AWG, consisting of Si photonic wire waveguides, was designed using the complete modeling \\ simulation in the beam propagation method. The device was fabricated on an SOI substrate and \\ evaluated in the wavelength range around $1.55 \mu \mathrm{m}$, with an effective area of $230 \times 160 \mu \mathrm{m}$. Clear \\ demultiplexing characteristics were observed with a channel spacing of $1.91 \mathrm{~nm}$. The influence of the \\ waveguide widths on crosstalk defined by adjacent channel crosstalk and phase error is discussed. \\ Insertion loss, crosstalk, and nonuniformity of loss were measured to be $-3.18,-23.1$, and $-1.35 \mathrm{~dB}$, \\ respectively. Thus, the AWG design is the best choice for a fiber Bragg grating demodulation \\ microsystem.

\section{Keywords} \\ waveguide, arrayed, silicon, channel, eight, compact, grating, optimized, practical, analysis, fabrication \\ Disciplines \\ Engineering | Science and Technology Studies

\section{Publication Details} \\ Li, H., Bai, Y., Dong, X., Li, E., Li, Y., Zhou, W. \& Liu, Y. (2013). Practical fabrication and analysis of an \\ optimized compact eight-channel silicon arrayed-waveguide grating. Optical Engineering, 52 (6), \\ 064602-1-064602-5.

\section{Authors} \\ Hongqiang Li, Yaoting Bai, Xiaye Dong, Enbang Li, Yang Li, Wenqian Zhou, and Yu Liu
}




\section{Optical Engineering}

\section{Practical fabrication and analysis of an optimized compact eight-channel silicon arrayed-waveguide grating}

Hongqiang Li

Yaoting Bai

Xiaye Dong

Enbang Li

Yang Li

Wenqian Zhou

Yu Liu 


\section{Practical fabrication and analysis of an optimized compact eight-channel silicon arrayed-waveguide grating}

\author{
Hongqiang Li \\ Yaoting Bai \\ Xiaye Dong \\ Tianjin Polytechnic University \\ School of Electronics and Information Engineering \\ Tianjin 300387, China \\ E-mail: lihongqiang@tjpu.edu.cn

\section{Enbang Li} \\ University of Sydney \\ Centre for Ultrahigh Bandwidth Devices for Optical \\ Systems \\ New South Wales 2006, Australia
}

\section{Yang Li \\ Wenqian Zhou \\ Yu Liu}

Tianjin Polytechnic University

School of Electronics and Information Engineering

Tianjin 300387, China

\begin{abstract}
We have designed, fabricated, and characterized a $1 \times 8$ ultrasmall compact arrayed-waveguide grating (AWG) on silicon-on-insulator (SOI) in a fiber grating demodulation integration microsystem. The miniature AWG, consisting of Si photonic wire waveguides, was designed using the complete modeling simulation in the beam propagation method. The device was fabricated on an SOI substrate and evaluated in the wavelength range around $1.55 \mu \mathrm{m}$, with an effective area of $230 \times 160 \mu \mathrm{m}$. Clear demultiplexing characteristics were observed with a channel spacing of $1.91 \mathrm{~nm}$. The influence of the waveguide widths on crosstalk defined by adjacent channel crosstalk and phase error is discussed. Insertion loss, crosstalk, and nonuniformity of loss were measured to be $-3.18,-23.1$, and $-1.35 \mathrm{~dB}$, respectively. Thus, the AWG design is the best choice for a fiber Bragg grating demodulation microsystem. @ 2013 Society of Photo-Optical Instrumentation Engineers (SPIE) [DOI: 10.1117/1.OE.52.6.064602]
\end{abstract}

Subject terms: arrayed-waveguide grating; silicon-on-insulator; microstructure fabrication; fiber grating demodulation integration microsystem.

Paper 130440 received Mar. 21, 2013; revised manuscript received May 14, 2013; accepted for publication May 24, 2013; published online Jun. 13, 2013.

\section{Introduction}

Optical fiber grating (FBG) sensor is an important type of optical passive component. This component is identified as the most competitive sensing element to constitute intelligent materials because of its unique advantages in the area of anti-electromagnetic interference, light-weightness, sensitivity, and so on. ${ }^{1}$ We have previously studied and demonstrated a wearable FBG-based sensor for human body temperature measurement in the form of intelligent clothing. ${ }^{2}$ We have also designed a new kind of FBG demodulation scheme, an arrayed-waveguide grating (AWG) demodulation integration microsystem (Fig. 1), characterized by high resolution, small-sized flexible design, and the capability to transmit multiple signals simultaneously. ${ }^{3}$

The production of an ultra compact AWG for a fiber Bragg grating demodulation integration microsystem is particularly important because the traditional AWG is large and expensive. The reported manufacturing materials for AWG are $\mathrm{SiO}_{2} / \mathrm{Si}$, silicon-on-insulator (SOI), InGaAsP/InP, polymer, $\mathrm{Si}_{3} \mathrm{~N}_{4}, \mathrm{SiO}_{n}$, and so on. ${ }^{4-6}$ SOI-based AWG has been extensively investigated ${ }^{7-9}$ because of its ultrahigh relative refractive index difference $\Delta$ in the Si core and low index claddings. These characteristics allow sharp bends, sizes in the micrometer scale, and easy-to-large scale integration of structure with other devices in the microsystem. However, these attributes also make the design and fabrication of such AWG difficult.

In this paper, we demonstrate an optimized design of SOIbased AWG with the central wavelength of $1550.918 \mathrm{~nm}$, which is a key component in AWG demodulation microsystems. To increase the coupling efficiency of fiber and AWG,

0091-3286/2013/\$25.00 @ 2013 SPIE the cone-shaped mold spot converter was used in the design process. The modal images from the fabricated AWG output waveguides were observed, and the performance of the design was analyzed by comparing simulation results experimentally in terms of insertion loss, crosstalk, and nonuniformity between the center and the outer channels.

\section{Theoretical Design and Studies}

Figure 2 shows the schematic diagram of an AWG structure consisting of the input and output waveguides as well as concave slab waveguide star couplers [or free propagation region (FPR)]. The components are connected by an array of waveguides with a constant path length difference and $\Delta L$ between adjacent waveguides. ${ }^{10}$

The parameters of the designed AWG were simulated using the beam propagation method and technology parameters which have guiding significance for optimizing the design of AWGs. These parameters include insertion loss, loss uniformity, crosstalk, and passband width, among others (Fig. 3). The results of the parameter optimization are shown in Table 1.

A reverse taper waveguide is used as a cone-shaped mold spot converter, such that waveguide width is tapered down from the values which designed to $180 \mathrm{~nm}$ and docks to optical fiber, and couples the chip to the fiber. As a result of the Goos-Hänchen phase shift effect of light in the dielectric waveguide, a smaller waveguide size results in a more powerful leakage optical mode, and a wider model results in a less complex and more effective approach to obtain effective indices of the optical fiber model and waveguide. Thus, this kind of taper can achieve very good mode matching and energy transfer. 


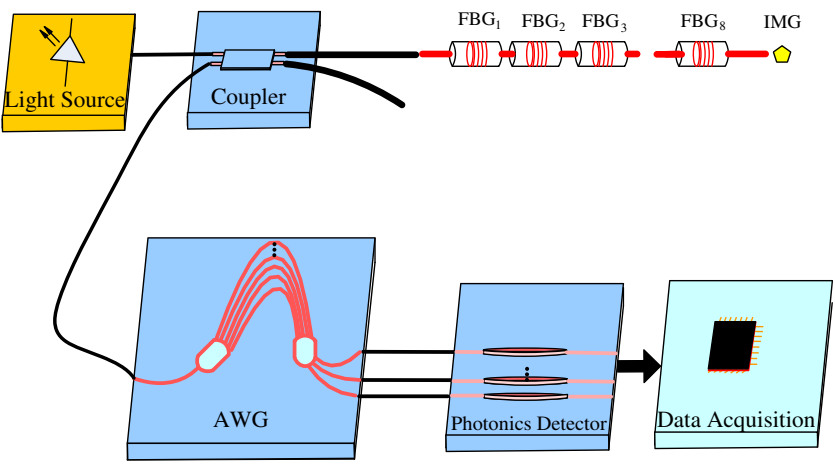

Fig. 1 Fiber grating demodulation system based on AWG.

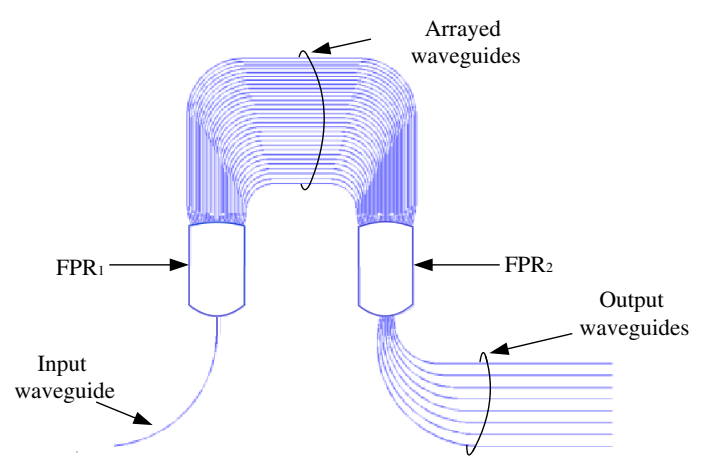

Fig. 2 Schematic top view of the AWG with geometrical design parameters.

\section{Fabrication and Characterization}

All the devices were fabricated on a $200 \mathrm{~mm}$ SOI wafer with a 220-nm thick Si guiding layer on top of a $2 \mu \mathrm{m}$ buried $\mathrm{SiO}_{2}$ layer. The minimum waveguide width of the designed $\mathrm{Si}$ nanowires AWG was only $0.34 \mu \mathrm{m}$ because its production feature size is in the nanometer level, which allows small existence process errors.

Electron beam lithography (EBL) technology was used to achieve the lithography of the AWG device because ordinary lithography technology was not applicable in the silicon
Table 1 Parameter optimization results of the SOI-based AWG.

\begin{tabular}{lc}
\hline \hline Parameters & Value \\
\hline Wavelength & $1550.918 \mathrm{~nm}$ \\
Number of output waveguides & 8 \\
Number of arrayed waveguides & 25 \\
Path length difference & $19.76 \mu \mathrm{m}$ \\
Length of free propagation region & $67 \mu \mathrm{m}$ \\
Diffraction order & 40 \\
Wavelength spacing & $2 \mathrm{~nm}$ \\
\hline \hline
\end{tabular}

nanowire AWG processing designed in this paper. Before the EBL exposure, approximately 110-nm thick polymethylmethacrylate-AR-P679.04 photoresist with high resolution, high contrast, and high transmittance rate of visible light was spin-coated on the chip. After prebaking, Raith150 EBL was used for exposure lithography. The exposure step was $10 \mathrm{~nm}$, and the writing field size was $300 \times 300 \mu \mathrm{m}$ during the exposure process. The compensation of graphical layout and regulation of the electronic beam dose was achieved simultaneously to ensure uniformity and smoothness of the width and sidewall of the AWG silicon nanowire waveguides. Then the development was completed. Hardening baking was performed to facilitate evaporation of the remaining photoresist solvent and improve the adhesiveness of SOI wafer surface which was achieved by the photoresist. Finally, exposure lithography of the AWG was completed.

Alcate601E plasma etching system was used to etch the AWG device waveguide. The deepest etching depth of the system was greater than $600 \mu \mathrm{m}$, and etching uniformity was less than $\pm 2.5 \%$. During ICP etching, $\mathrm{CHF}_{3}$ and $\mathrm{O}_{2}$ were chosen as the etching gases. To ensure a high-level etching rate and prevent accumulation of SOI surface sediment during the Si etching process, we set the following



Fig. 3 Schematic representation of technology parameters. 


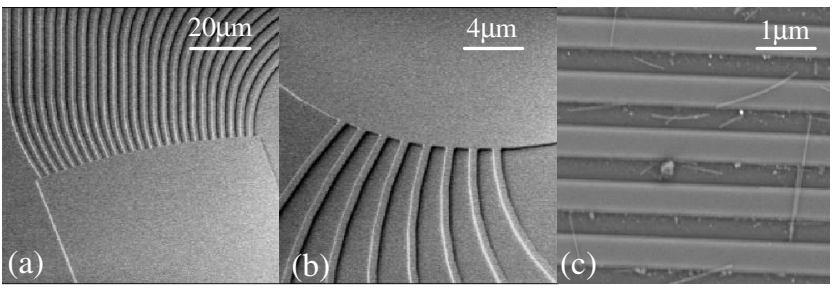

Fig. 4 SEM images of the fabricated AWG: (a) array, (b) output, and (c) straight waveguides.

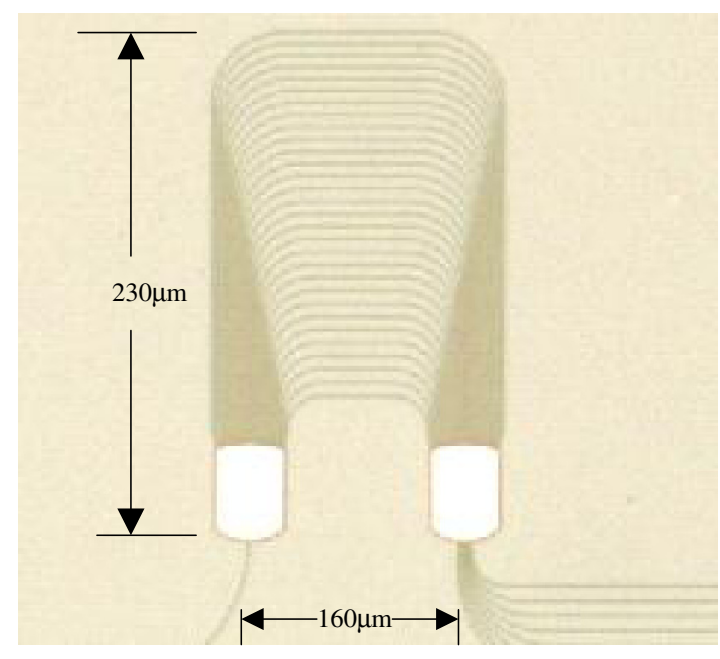

Fig. 5 Chip of fabricated AWG.

parameters: etching source power, $600 \mathrm{~W}$; bias power, $100 \mathrm{~W} ; \mathrm{CHF}_{3}$ flow, 100 scem; $\mathrm{O}_{2}$ flow, 10 scem; and pressure of the etching reaction chamber was less than $0.1 \mathrm{~Pa} . \mathrm{Si}$ etching was completed at $0.01 \mu \mathrm{m} / \mathrm{min}$. At the same time, helium was used to cool the etching reaction chamber. After about 20 min, a $0.22-\mu$ m thick Si layer was finished. AWG manufacture was completed after cutting and end face polishing. Figure 4 illustrates the chip of fabricated eight-channel AWG using BX51M microscope of OLYMPUS and Fig. 5 illustrates the scanning-electron microscope (SEM) images of the fabricated AWG. The images show that the AWG has good structure and well-defined sidewalls and corners.

\section{Results and Discussion}

During the test, to improve coupling efficiency, taper-type optical fibers are used, as shown in Fig. 6; the ASE broadband high stable light source and a spectrometer are used. In this section, first we describe the effect of the width of waveguides used in the waveguide array on the AWG spectral

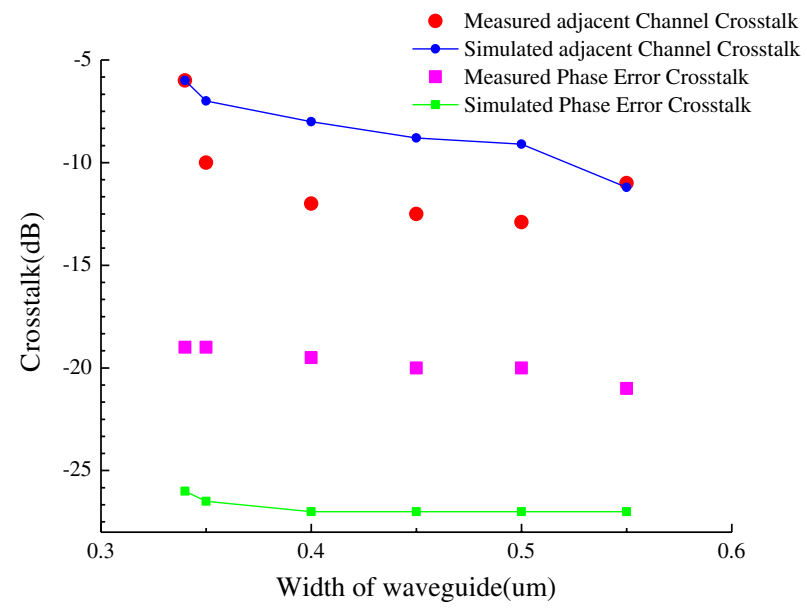

Fig. 7 Crosstalk versus width of waveguides.

response and next we discuss the characterization of AWG optimized based on the previous results.

\subsection{Waveguide Width Optimization}

The waveguide structure is the basis of the plane waveguide device. A good design ensures the excellent transmission of light and device performance. To map the influence of the waveguide width, we designed AWGs with widths of $0.34,0.35,0.4,0.45,0.5$, and $0.55 \mu \mathrm{m}$ [all widths guarantee single-mode transmission for transverse electric (TE) mode]. In Fig. 7, we can observe that the experimental phase errors follow the same trend as the simulated results. The phase errors are mostly dependent on the number of waveguides used in the waveguide array. The increased effect of waveguide width corresponds to a small phase error effect. The change in the experimental phase errors is greater than that of the simulated phase errors. This observation is attributed to the sidewall roughness and thickness variations in the waveguides. In Fig. 7, we can also observe that the effect of adjacent channel crosstalk increases for AWG waveguides with greater width. Experimental adjacent channel crosstalk does not match with the simulation. This discrepancy is ascribed to the thickness variation and inclined sidewall of the waveguide in reality.

\subsection{Optimized Spectral Response}

We can conclude from the previous results that the $1 \times 8$ AWG with $0.4-\mu \mathrm{m}$ wide waveguide presents a good compromise in achieving good spectral response with good crosstalk level and small size. Using our AWG model, we simulated this device in more detail. Figure 8 illustrates that the device
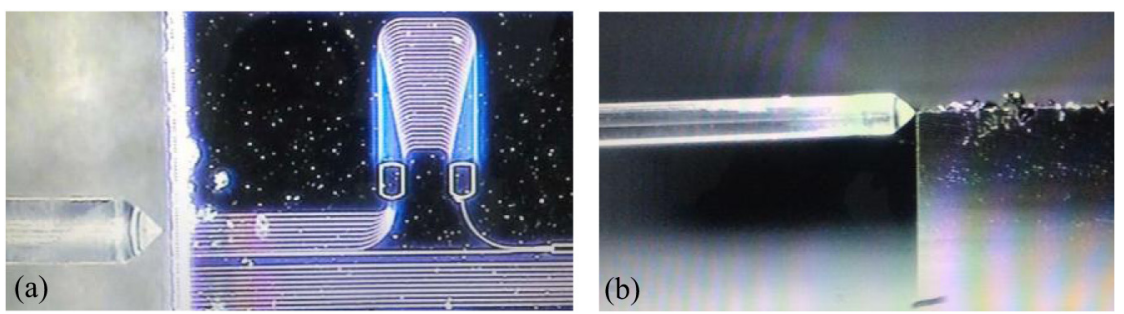

Fig. 6 Alignment of the taper-type optical fiber and waveguide: (a) top view and (b) front view. 


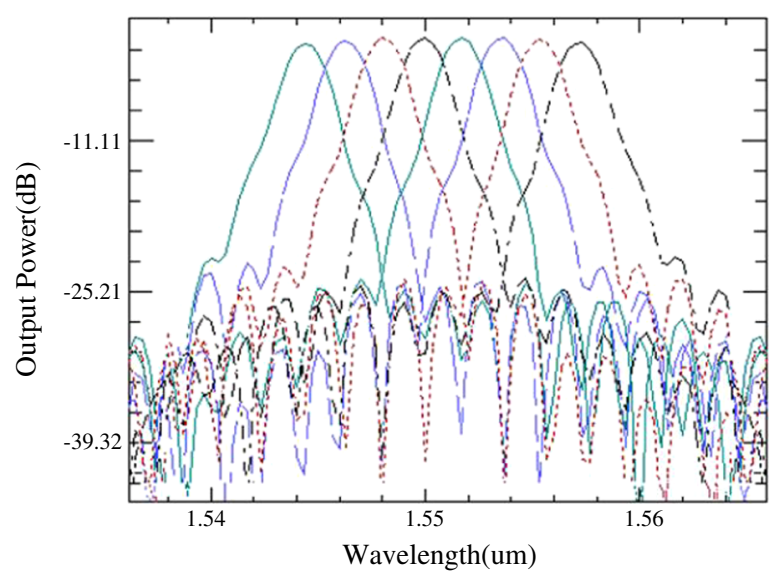

Fig. 8 Simulation result of AWG.

is expected to exhibit a central channel loss of $-2.15 \mathrm{~dB}$, an insertion loss nonuniformity of $0.8 \mathrm{~dB}$, and a crosstalk level of $-25.5 \mathrm{~dB}$. Experimentally, we find (Fig. 9) a central channel loss of $-3.18 \mathrm{~dB}$, an insertion loss nonuniformity of $-1.34 \mathrm{~dB}$, and a crosstalk of $-23.1 \mathrm{~dB}$, defined by the phase error and adjacent channel crosstalk level. AWG output spot is collected and displayed by an infrared camera (Fig. 10). The eight channel spots are clear and uniform.

The difference in the center channel loss between the simulated and experimental results is caused by the waveguide propagation loss, slab propagation loss, and a propagation angle of the aperture mismatch between the designed and the fabricated AWGs. The mismatch of insertion loss nonuniformity resulted from the dispersion mismatch and the aperture field profile mismatch between the designed and fabricated waveguides. One of the main concerns for this type of device is the nonuniformity of bandwidth and channel spacing over the different channels caused by the dispersion mismatch between the designed and fabricated waveguides. Figure 11 illustrates the fiber-to-fiber measurement of the device and the reference waveguide. Figure 11 shows that the envelope of the device spectrum follows the envelope of the reference waveguide spectrum. We observe



Fig. 9 Measurement result of $8 \times 250 \mathrm{GHz}$ AWG.

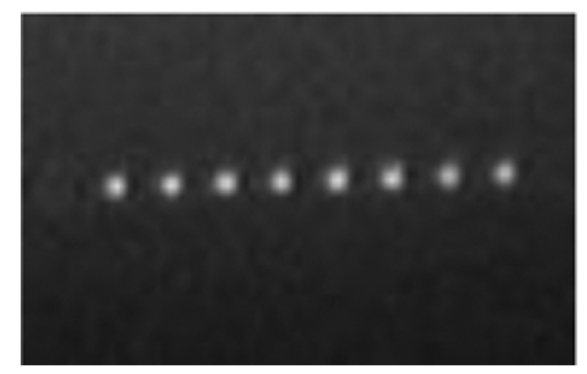

Fig. 10 Output infrared light spot.



Fig. 11 Fiber-to-fiber measurement of AWG and the reference waveguide.

that for this device [Fig. 12(a)], the nonuniformity of the 1 and $3 \mathrm{~dB}$ bandwidths is 0.074 and $0.046 \mathrm{~nm}$, respectively. Each channel center wavelength is shown in Fig. 12(b) with the best-fitting straight line slope of 1.91471. The channel spacing is consistent with the design value. This deviation is acceptable, considering the mismatch in the dispersion relation and waveguide thickness used in our model compared with the actual fabricated waveguide.

\section{Conclusion}

We have demonstrated a $230 \times 160 \mu \mathrm{m}$ SOI-based AWG with a channel spacing of $2 \mathrm{~nm}$, a crosstalk of $-23.1 \mathrm{~dB}$, an insertion loss of -3.18 , and nonuniformity of loss of $-1.35 \mathrm{~dB}$. The average channel spacing obtained from the experiment is $1.91 \mathrm{~nm}$, which depicts good wavelength uniformity. The experimental results match well with the simulation results. To further reduce crosstalk, the array waveguide width consistency and optimization of process preparation should be improved. The adjacent channel crosstalk depends on the waveguide width and matches incompletely with the simulations. The nonuniformity of the bandwidths is limited and given by 0.074 and $0.046 \mathrm{~nm}$ for the 1 and $3 \mathrm{~dB}$ bandwidths, respectively. Compared with the performance of the state-of-the-art, ${ }^{9,11}$ our proposed design has bigger but still small sizes, a smaller crosstalk, and a better transmission spectra. These results demonstrate that we are able to design and fabricate compact silicon AWGs with predictable spectral response for a fiber Bragg grating demodulation microsystem. 

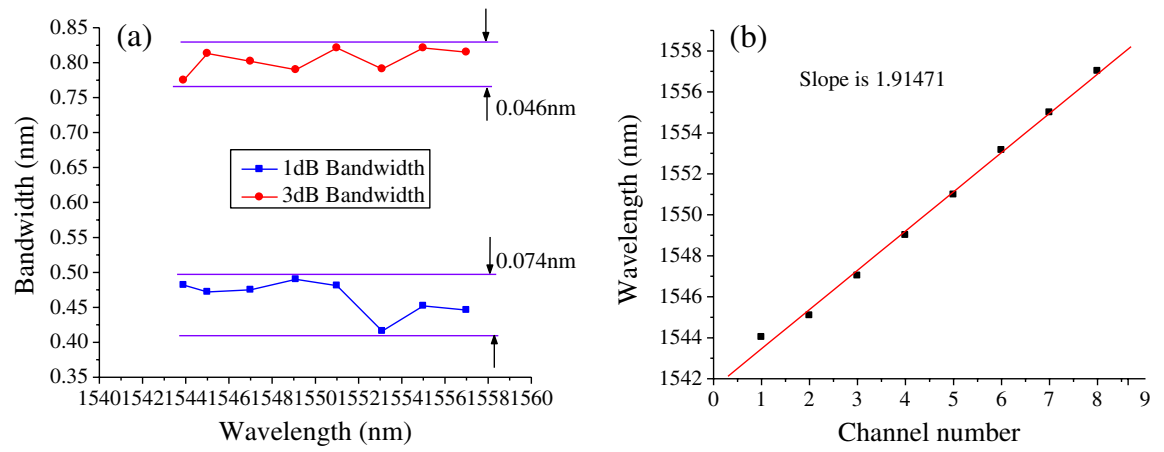

Fig. 12 (a) Associated nonuniformity of 1 and $3 \mathrm{~dB}$ bandwidth and (b) the central wavelength in each output port.

\section{Acknowledgments}

This work is supported by the National Natural Science Foundation of China (No. 61177078, 60877049) and the Specialized Research Fund for the Doctoral Program of Higher Education of China (No. 20101201120001).

\section{References}

1. T. Zhu et al., "Fabry-Perot optical fiber tip sensor for high temperature measurement," Opt. Commun. 283(19), 3683-3685 (2010).

2. H. Li et al., "Wearable sensors in intelligent clothing for measuring human body temperature based on optical fiber Bragg grating," Opt. Express 20(11), 11740-11752 (2012).

3. X. Yu, C. Miao, and H. Li, "Research on human body temperature measurement models of intelligent clothing based on optical fiber Bragg grating," Opt. Tech. 37(6), 704-708 (2011) (in Chinese).

4. A. Sugtia et al., "Very low insertion loss arrayed waveguide grating with vertically tapered waveguides," IEEE Photon. Technol. Lett. 12(9), 1180-1182 (2000).

5. Y. Nasuhi et al., "Design and fabrication of a broadband polarization and temperature insensitive arrayed waveguide grating on InP,' Opt. Express 13(14), 5535-5541 (2005).

6. D. Dai et al., "Low-loss $\mathrm{Si}_{3} \mathrm{~N}_{4}$ arrayed-waveguide grating (de)multiplexer using nano-core optical waveguides," Opt. Express 19(15), 14130-14136 (2011).

7. P. Cheben et al., "A high-resolution silicon-on-insulator arrayed waveguide grating microspectrometer with submicrometer aperture waveguides," Opt. Express 15(5), 2299-2306 (2007).

8. L. Zhao et al., "Design and fabrication of an 8 channel, and $1.6 \mathrm{~nm}$ arrayed waveguide grating based on Si nanowires," J. Optoelectron. Laser 21(11), 1589-1592 (2010) (in Chinese).

9. X. Fu and D. Dai, "Ultra-small Si-nanowire-based $400 \mathrm{GHz}$ spacing $15 \times 15$ arrayed-waveguide grating router with microbends," Electron. Lett. 47(4), 266-268 (2011).

10. H. Li et al., "Design of $1 \times 8$ silicon nanowire arrayed waveguide grating for on-chip arrayed waveguide grating demodulation integration microsystem," Opt. Eng. 51(12), 1230011 (2012).

11. K. Sasaki et al., "Arrayed waveguide grating of $70 \mu \mathrm{m} \times 60 \mu \mathrm{m}$ size based on $\mathrm{Si}$ photonic wire waveguides," Electron. Lett. 41(14), 801-802 (2005).

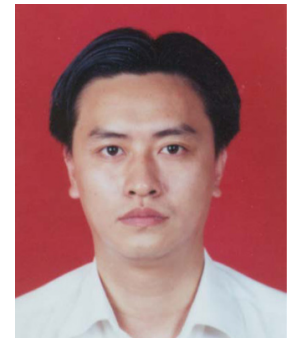

Hongqiang Li received his $\mathrm{BE}, \mathrm{MSc}$, and PhD degrees from Tianjin Polytechnic University. He is an assistant professor in the School of Electronics and Information Engineering, Tianjin Polytechnic University, China. His research interests lie in the area of optical fiber grating sensor demodulation and optoelectronics integration.

Yaoting Bai is working toward a master's degree in the School of Electronics and Information Engineering, Tianjin Polytechnic University China.

Xiaye Dong is working toward a master's degree in the School of Electronics and Information Engineering, Tianjin Polytechnic University China.

Enbang Li is a professor at the Centre for Ultrahigh bandwidth Devices for Optical Systems (CUDOS), The University of Sydney Australia.

Yang Li is working toward a master's degree in the School of Electronics and Information Engineering, Tianjin Polytechnic University China.

Wenqian Zhou is working toward a master's degree in the School of Electronics and Information Engineering, Tianjin Polytechnic University, China.

Yu Liu is working toward a master's degree in the School of Electronics and Information Engineering, Tianjin Polytechnic University China. 